\title{
Annual Incidence and Severity of Acute Episodes in Hereditary Thrombotic Thrombocytopenic Purpura
}

Tracking no: BLD-2020-009801R1

Erika Tarasco (Department for BioMedical Research, University of Bern, Bern, Switzerland) Lukas Bütikofer (CTU Bern, ) Kenneth Friedman (Division of Hematology and Oncology, Medical College of Wisconsin, United States) James George (University of Oklahoma Health Sciences Center, United States) Ingrid Hrachovinova (Institute of hematology and blood transfusion, Czech Republic) Paul Knöbl (Vienna Medical University, Austria) Masanori Matsumoto (Nara Medical University, Japan) Anne Sophie von Krogh (Department of Clinical and Molecular Medicine, faculty of Medicine and Health Sciences, NTNU, Trondheim, Norway) Isabella Aebi-Huber (Department for BioMedical Research, University of Bern, Bern, Switzerland) Zuzana Cermakova (Blood Center, ) Magdalena Górska-Kosicka (Institute of Hematology and Transfusion Medicine, Poland) Katarzyna Jalowiec (Department of Hematology and Central Hematology Laboratory, Switzerland) Carlo Largiadèr (Bern University Hospital and University of Bern, Switzerland) Zoltan Prohaszka (Semmelweis University, Hungary) György Sinkovits (Semmelweis University, Hungary) Jerzy Windyga (Institute of Haematology and Transfusion Medicine, Poland) Bernhard Lämmle (Center for Thrombosis and Hemostasis, University Medical Center Mainz, Mainz, Germany, Germany) Johanna Kremer Hovinga (Department for BioMedical Research, University of Bern, Bern, Switzerland)

\section{Abstract:}

Hereditary thrombotic thrombocytopenic purpura (hTTP) is a rare thrombotic microangiopathy characterized by severe congenital ADAMTS13 deficiency and recurring acute episodes causing morbidity and premature death. Information on the annual incidence and severity of acute episodes in hTTP patients is largely lacking.

This study reports prospective data of 87 patients from the Hereditary TTP Registry (ClinicalTrials.gov NCT01257269) for survival, frequency and severity of acute episodes from enrollment until December 2019.

The 87 patients, followed for median 4.2 years (range 0.01-15), had a median age at overt disease onset and at clinical diagnosis of 4.6 years and of 18 years (range 0.0-70 for both), respectively. Fortythree patients received regular plasma prophylaxis, while 22 did not, and treatment changed over time or was unknown in the remaining 22. Forty-three patients experienced 131 acute episodes of which 91 (69\%) occurred in patients on regular prophylaxis. This resulted in an annual incidence of acute episodes of 0.36 (95\%CI 0.29-0.44) with and of 0.41 (95\%CI 0.30-0.56) without regular plasma treatment. More than one third of acute episodes $(n=51)$ were documented in children <10 years of age at enrollment and were often triggered by infections. Their annual incidence of acute episodes was significantly higher than in patients $>40$ years of age (1.18 [95\% CI 0.88-1.55] vs. 0.14 [95\% CI 0.08-0.23]).

Prophylactic plasma infusion regimens used were insufficient to prevent acute episodes in many patients. Such regimens are burdensome, caregivers, patients and their guardians are reluctant to start regular plasma infusions, from which particularly children would benefit.

Conflict of interest: COI declared - see note

COI notes: PK received consultancy and advisory board fees, speaker honoraria and travel grants from Ablynx/Sanofi, Alexion, Shire/Takeda. M.M. is a member of advisory board of Takeda, Alexion, and Sanofi. A.S. V. K. is a member of advisory board of Sanofi Genzyme J.W. received grant support or lectures honoraria from Alexion, Alnylam Pharmaceuticals, Baxalta, CSL Behring, Ferring Pharmaceuticals, Novo Nordisk, Octapharma, Rigel Pharmaceuticals, Roche, Sanofi/ Genzyme, Shire/Takeda, Sobi, Werfen. B.L. is chairman of the data monitoring safety committees for the Baxalta 281102 study (rADAMTS13 in congenital TTP) and for the Takeda SHP655-201 study (rADAMTS13 in acquired TTP), now both run by Takeda. He is a member of the advisory board Ablynx, now part of Sanofi for the development of caplacizumab. He received congress travel support and/or lecture fees from Baxter, Ablynx, Alexion, Siemens, Bayer, Roche and Sanofi. J.A.K.H. is a member of the advisory board of Shire, member of the Takeda group of companies for the development of rADAMTS13; and of Ablynx, now part of Sanofi for the development of caplacizumab. The other authors have nothing to disclose.

Preprint server: No;

Author contributions and disclosures: ET, LB, BL, JAKH designed the study. ET wrote the paper. ET, IAH, 
KAJ collected data. ET, LB, BL and JAKH analyzed data. KDF, JNG, IH, PNK, MM, ASVK, BL and JAKH approved the research project and helped editing. All authors revised the manuscript.

Non-author contributions and disclosures: No;

Agreement to Share Publication-Related Data and Data Sharing Statement: emails to the corresponding author

Clinical trial registration information (if any): NCT01257269, observational study 


\section{Annual Incidence and Severity of Acute Episodes in Hereditary Thrombotic Thrombocytopenic Purpura}

Erika Tarasco ${ }^{1,2}$, Lukas Bütikofer ${ }^{3}$, Kenneth D. Friedman ${ }^{4}$, James N. George ${ }^{5}$, Ingrid Hrachovinova ${ }^{6}$, Paul N. Knöbl ${ }^{7}$, Masanori Matsumoto ${ }^{8}$, Anne Sophie von Krogh ${ }^{9,10}$, Isabella Aebi-Huber ${ }^{1,2}$, Zuzana Cermakova $^{11}$, Magdalena Górska-Kosicka ${ }^{12}$, Katarzyna A. Jalowiec ${ }^{1}$, Carlo R. Largiadèr ${ }^{13}$, Zoltán Prohászka $^{14}$, György Sinkovits ${ }^{14}$, Jerzy Windyga ${ }^{12}$, Bernhard Lämmle ${ }^{1,15}$, Johanna A. Kremer Hovinga ${ }^{1,2}$

${ }^{1}$ Department of Hematology and Central Hematology Laboratory, Inselspital, Bern University Hospital, Bern, Switzerland

${ }^{2}$ Department for BioMedical Research, University of Bern, Bern, Switzerland

${ }^{3}$ CTU Bern, University of Bern, Bern, Switzerland

${ }^{4}$ Division of Hematology and Oncology, Medical College of Wisconsin, Milwaukee, Wisconsin, USA

${ }^{5}$ Department of Biostatistics \& Epidemiology, College of Public Health, University of Oklahoma Health Sciences Center, Oklahoma City, OK, USA

${ }^{6} \mathrm{NRL}$ for Hemostasis, Institute of Hematology and Blood Transfusion, Prague, Czech Republic

${ }^{7}$ Division of Hematology and Hemostasis, Department of Medicine 1, Medical University of Vienna, Austria

${ }^{8}$ Department of Blood Transfusion Medicine, Nara Medical University, Kashihara, Japan

${ }^{9}$ Department of Hematology, St Olavs Hospital, Trondheim University Hospital, Trondheim, Norway,

${ }^{10}$ Department of Clinical and Molecular Medicine, faculty of Medicine and Health Sciences, NTNU, Trondheim, Norway

${ }^{11}$ Blood Center, University Hospital Ostrava, Ostrava, Czech Republic

12 Department of Haemostasis Disorders and Internal Medicine Institute of Hematology and Transfusion Medicine, Warsaw, Poland

${ }^{13}$ University Institute of Clinical Chemistry, Inselspital, Bern University Hospital, Bern, Switzerland

${ }^{14}$ Research Laboratory, Department of Internal Medicine and Hematology, and Research Group for Immunology and Hematology, Eötvös Loránd Research Network and Semmelweis University, Budapest, Hungary

${ }^{15}$ Center for Thrombosis and Hemostasis, University Medical Center Mainz, Mainz, Germany

Correspondence:

Johanna A. Kremer Hovinga, MD

Department of Hematology and Central Hematology Laboratory, Bern University Hospital, Inselspital, CH-3010 Bern, Switzerland

Phone: +41316320265

johanna.kremer@insel.ch 


\section{KEY POINTS}

1. Annual incidence of acute episodes is highest in children $<10$ years of age and decreases with increasing age

2. Currently applied plasma prophylaxis is often not sufficient to prevent the occurrence of acute episodes.

\section{ABSTRACT}

Hereditary thrombotic thrombocytopenic purpura (hTTP) is a rare thrombotic microangiopathy characterized by severe congenital ADAMTS13 deficiency and recurring acute episodes causing morbidity and premature death. Information on the annual incidence and severity of acute episodes in hTTP patients is largely lacking.

This study reports prospective data of 87 patients from the Hereditary TTP Registry (ClinicalTrials.gov NCT01257269) for survival, frequency and severity of acute episodes from enrollment until December 2019.

The 87 patients, followed for median 4.2 years (range $0.01-15$ ), had a median age at overt disease onset and at clinical diagnosis of 4.6 years and of 18 years (range $0.0-70$ for both), respectively. Forty-three patients received regular plasma prophylaxis, while 22 did not, and treatment changed over time or was unknown in the remaining 22. Forty-three patients experienced 131 acute episodes of which 91 (69\%) occurred in patients on regular prophylaxis. This resulted in an annual incidence of acute episodes of $0.36(95 \% \mathrm{Cl} 0.29$ $0.44)$ with and of $0.41(95 \% \mathrm{Cl} 0.30-0.56)$ without regular plasma treatment. More than one third of acute episodes $(n=51)$ were documented in children $<10$ years of age at enrollment and were often triggered by infections. Their annual incidence of acute episodes was significantly higher than in patients $>40$ years of age $(1.18[95 \% \mathrm{Cl} 0.88-1.55]$ vs. $0.14[95 \%$ Cl 0.08-0.23]). 
Prophylactic plasma infusion regimens used were insufficient to prevent acute episodes in many patients. Such regimens are burdensome, caregivers, patients and their guardians are reluctant to start regular plasma infusions, from which particularly children would benefit. 


\section{INTRODUCTION}

Hereditary thrombotic thrombocytopenic purpura (hTTP), also known as Upshaw-Schulman syndrome (OMIM: 274150), is a rare thrombotic microangiopathy characterized by severe congenital ADAMTS13 deficiency and smoldering but also acute disease episodes with thrombocytopenia, hemolytic anemia and microvascular thrombosis leading to end-organ damage (mainly affecting brain and kidneys). ${ }^{1-3}$ ADAMTS13 is essential for cleaving hemostatically very active, ultra-large von Willebrand factor (vWF) multimers into less sticky regular sized multimers to prevent spontaneous platelet adhesion and eventually the formation of vWF-platelet rich thrombi occluding the microcirculation. ${ }^{1-3}$ Inheritance of hTTP is autosomal recessive and today more than 150 causative ADAMTS13 mutations have been reported..$^{4-8}$ Hereditary TTP has an estimated prevalence of $0.5-2$ cases per one million, ${ }^{3}$ although the prevalence may be considerably higher in certain areas. ${ }^{9}$

Even with identical ADAMTS13 genotypes, hTTP patients manifest with variable disease onset and exhibit heterogeneous clinical courses. ${ }^{7-10}$ While some patients seemingly remain without disease episodes far into adulthood, others require exchange blood transfusion in the first days of life and/or experience already numerous acute TTP episodes in childhood. ${ }^{7-12}$ Although hTTP can become overt at any time during a patient's life, ${ }^{3,7,8}$ early childhood and pregnancy are periods of high risk of manifestation. ${ }^{11-17}$

Acute TTP episodes in hTTP occur spontaneously, or are triggered by factors associated with increased vWF plasma levels, endothelial cell activation or increased shear stress, such as a patent ductus arteriosus, ${ }^{18}$ infections, ${ }^{5,8,12,17}$ pregnancy, ${ }^{5,8,13-16}$ alcohol $^{5,8}$ or certain drugs (desmopressin). ${ }^{5,19}$ The high number of hTTP patients having suffered from arterial thrombotic events already at relatively young age demonstrates the significance of acute episodes. ${ }^{20}$ At enrollment into the International Hereditary TTP Registry 25/120 (21\%) patients reported to have had a stroke, five patients a transient ischemic attack (TIA), and another five a myocardial infarction. ${ }^{8}$ Pre-existing arterial thrombotic events were present in 
more than $50 \%$ of hTTP patients enrolled at age $40-50$ years and over. While in the UK hereditary TTP cohort no myocardial infarction was observed, 14/73 (19\%) of patients had suffered from a stroke. ${ }^{7}$ Regular plasma infusions, usually administered in fortnightly intervals, are effective in preventing acute TTP episodes in individual patients over years. ${ }^{5,7}$, 8, 21, 22 Prophylactic plasma therapy is associated with regular visits at treatment centers, is time consuming and allergic reactions to plasma products are frequent; the decision to initiate such a regimen is not easily taken. Still, at enrollment into the International Hereditary TTP Registry $71 \%$ of hTTP patients were on regular plasma prophylaxis, ${ }^{8}$ a similar number of $69 \%$ was reported for the UK cohort. $^{7}$

At present, there are neither data on the annual incidence of acute episodes in hTTP patients, nor on the frequency and severity of episodes in patients on regular plasma prophylaxis, nor is there information on the long-term advantage of this burdensome treatment.

We addressed these questions by investigating the occurrence, the severity of acute disease episodes and survival as well as treatment over time in hTTP patients in the International Hereditary TTP Registry based on prospective follow-up data from patients' enrollment until their last scheduled visit by the end of 2019. 


\section{MATERIAL AND METHODS}

The Hereditary TTP Registry (www.ttpregistry.net; [NCT01257269]) is an ambi-directional cohort study for patients diagnosed with hereditary TTP and their family members. ${ }^{8,23}$ The Kantonale Ethik-Kommission Bern, as lead ethics committee (EC), approved the study in September 2006 (study number 031/06). Before enrollment of patients, each participating site obtained approval from its local or national institutional review board (IRB) or EC. Due to regulatory obligations, renewal of EC approval at all Japanese sites is underway, and all Japanese Registry participants have to undergo re-consenting, which is pending in 44 of them. These 44 patients were consequently not included in this follow-up analysis.

At the end of 2012, paper case report forms (CRFs) were replaced by web-based, electronic CRFs with associated database (webspirit $\AA$, 2mt software, Ulm, Germany). This enables automatic e-mail reminders of upcoming follow-up visits as well as the sites to enter new data directly after personalized login. Central project coordination including data monitoring and cleaning is done at Bern University Hospital.

\section{Patients and Data collection}

For this first prospective study, confirmed hTTP patients ${ }^{8}$ having an enrollment date before August $1^{\text {st }}, 2018$ and at least one follow-up visit, or a study-end visit by the end of 2019 were considered eligible. A follow-up visit was defined as any visit, face-to-face or by phone call that happened after enrollment. Per study protocol, prospective follow-up visits were to be performed annually, and at occurrence of any acute episode. Not all patients were followed annually, although adherence improved during recent years. The mean and maximal gap between follow-up visits was 0.95 years (range 0.01-5.6 years) and 1.6 years (range 0.01-8.2 years), respectively, with the majority of patients $(n=54)$ having a maximal gap of 1-2 years between follow-up visits (Supplemental Table 1).

We analyzed the following data: course of hTTP since enrollment, incidence and severity of acute episodes; survival; treatment including regular plasma therapy and changes thereof, 
intake of antihypertensive, antiplatelet and/or anticoagulant drugs; treatment side effects; and pregnancies.

To calculate a patient's annual volume of plasma received for prophylaxis, we set the volume of unit of solvent detergent plasma as $200 \mathrm{ml}$ (standardized volume of Octaplas, the most widely used solvent detergent plasma in our cohort), and of fresh frozen plasma as $250 \mathrm{ml}$ (the volume of one unit of fresh frozen plasma typically ranges from 200-300ml). In the absence of information from the Center caring for the respective patient, we assumed that the number of plasma units administered per session, the treatment interval and body weight were stable between two visits.

\section{Specification and severity of an acute TTP episode}

An acute TTP episode was defined as a typical TTP episode ${ }^{24}$ or an acute cerebrovascular or cardiovascular or other ischemic event or a renal illness for which the patient required and/or received medical care in addition to the patient's regular prophylactic treatment, e.g. with plasma infusion. In addition, a maternal pregnancy complication resulting in pregnancy termination (abortion or delivery) after 10 and before 34 weeks of gestation was considered an acute episode (Table 1). We defined four categories to grade the severity of the acute TTP episode, with category 1 characterized by mild clinical manifestations; categories 2 and 3 by moderate to severe clinical symptoms and signs; and category 4 by fatal outcome (Table 1). Episodes were scored by the Registry project manager (E.T.) and at least one Registry steering committee member (B.L. and/or J.A.K.H) independently. In case of discordant scores, the hTTP episodes in question were discussed again with the treating physician and then the whole Registry team at Bern University Hospital until a consensus was reached.

\section{ADAMTS13 parameters}

In 70/87 patients, ADAMTS13 activity was determined at Bern University Hospital, University of Bern, Switzerland by the modified VWF73-FRETS assay. ${ }^{25,26}$ In the remaining patients, 
ADAMTS13 activity was assessed in other laboratories employing various assay methods $(n=15)$, and not reported for two patients.

To assess a patient's baseline ADAMTS13 activity reflecting endogenous ADAMTS13 production, we considered only blood samples withdrawn at least 14 days after the last administration of plasma-containing blood products.

Functional ADAMTS13 inhibitors (positive results $>0.4 \mathrm{BU} / \mathrm{ml}$ ), anti-ADAMTS13 antibodies by ELISA (Technoclone ${ }^{\circledR}$; positive result $>15 \mathrm{AU} / \mathrm{ml}$ ) and genetic analysis of ADAMTS13 were performed at Bern University Hospital, as previously described. ${ }^{8,9}$

\section{Statistical analysis}

Categorical variables are reported as numbers and percentages, and compared between groups with Fisher's exact test. Continuous data are reported as median and range (and $25^{\text {th }}$ and $75^{\text {th }}$ percentiles for certain variables of interest), and compared by Wilcoxon rank-sum tests.

Incidence rates are presented with exact Poisson 95\% confidence intervals $(\mathrm{Cl})$. As it was not possible to follow-up all patients annually, we assessed the influence of large gaps between follow-up visits (that could lead to an underreporting of episodes) on the annual incidence rate estimates by a series of sensitivity analyses. We assumed a patient was lost to follow-up in case of gaps between visits of more than two, three and five years, respectively (Supplemental Table 2). Since the trend of results indicated that larger gaps were not affecting our results, we included all 87 confirmed hTTP patients in our analysis irrespective of their differences in follow-up years.

The effect of age at enrollment, gender, use of prophylaxis and follow-up time on the incidence of TTP episodes was analyzed using mixed-effects Poisson regression with robust standard errors based on the Huber-White sandwich estimator.

We used Stata version 15 (StataCorp. 2017. Stata Statistical Software: Release 14. College Station, TX) for all statistical analyses. 


\section{RESULTS}

\section{Patients overview: clinical and biochemical characteristics}

At the end of December 2019, the Hereditary TTP Registry had 209 participants of which 139 were confirmed hTTP patients (Figure 1). Five confirmed hTTP patients had died before inclusion into the Registry, three patients were lost to follow-up, and 44 Japanese patients were temporarily suspended because of regulatory reasons. Hence, $87 \mathrm{hTTP}$ patients (46 male and 41 female patients) contributed to this follow-up study (Figure 1). Their median follow-up time was 4.2 years (range $0.01-15$ years) and they had median three follow-up visits (range 1-10).

Most patients were Caucasians ( $n=75 ; 86 \%)$, followed by Asians $(n=6 ; 6.9 \%)$, Hispanics and others (each $n=3 ; 3.4 \%$ ). Nine patients had consanguineous parents, and 13 had a family member that was also affected by hTTP (one parent-child relation, the others were siblings). Further demographic and clinical features as well as treatments are listed in Table 2.

The median age at overt disease onset of the $87 \mathrm{hTTP}$ patients was 4.6 years, at clinical diagnosis 18 years (range for both $0.0-70$ years), at enrollment into the Registry and at last follow-up 26 years (range 1.2-75 years) and 32 years (range 2.1-79 years), respectively (Table 2). Patients who experienced acute episodes during prospective follow-up had a similar age at disease onset but a slightly, though statistically not significant, younger age at clinical diagnosis than those without events (15 [range 0.0-68] years vs. 20 [range 0.0-70] years, $p=0.12$ ) (Table 2). Overall, 36 patients had a history of neonatal hyperbilirubinemia, 22 of ischemic strokes and 13 of TIAs, eight patients were on dialysis and two had received a kidney transplant. Of note, preexisting conditions at enrollment (strokes, epileptic seizures) were more common in patients without acute episodes during follow-up (Table 2).

Forty-three patients were homozygous and 44 were compound heterozygous ADAMTS13 mutation carriers. ADAMTS13 activity was $\leq 10 \%$ in all $87 \mathrm{hTTP}$ patients, in 70 of them ADAMTS13 activity was assessed in the laboratory in Bern. Even though ADAMTS13 functional inhibitors were negative in all 77 patients tested, 7 out of $68(10.3 \%)$ patients 
tested had a positive anti-ADAMTS13 antibody ELISA result with antibody titers between 16.2 and 33.8 AU/mL (data not shown).

Occurrence of acute episodes during follow-up overall and in relation to age, sex and plasma prophylaxis

Figure 2 shows the complete overview of prospective follow-up from enrollment until their last scheduled visit until the end of December 2019 in the 87 confirmed hTTP patients. Patients are sorted according to their sex, prospective observation time, their prophylactic treatment and changes thereof. In addition, we show the occurrence of acute TTP episodes. Fortythree $(49.4 \%$ of the whole cohort) patients were always on regular prophylactic treatment, while $22(25 \%)$ had no prophylactic plasma treatment during follow-up. Eighteen patients received intermittent prophylaxis, including two women who were on regular plasma infusions only during pregnancies (Figure 2, patients \#76 and \#77). For four patients (Figure 2; patients \#84-87) details about their prophylactic regimen were not available.

In the insert (Figure $2 \mathrm{~B}$ ), the 25 patients aged $<18$ years (children) at enrollment are shown, sorted according to their age at enrollment. While ten children had no acute episode during prospective follow-up, the other 15 experienced 75 of the 131 (57.3\%) documented acute episodes. Patients \#1, 15, 16, 18, 27, 67, 69 and 74 suffered from multiple acute episodes despite undergoing regular plasma prophylaxis, indicating that the chosen and/or supportable regimens were insufficient (Figure 2B).

The 87 confirmed hTTP patients had 371 person-years of prospective follow-up. During follow-up, 44 patients (17 females) had no acute TTP episode, while the other 43 patients (24 females) experienced 131 acute episodes altogether (Table 3). This resulted in an overall annual incidence rate of acute episodes of 0.35 per person-year ( $95 \% \mathrm{Cl} 0.29-0.42)$. The annual incidence rates of patients on or not on regular plasma prophylaxis were similar (Table 3, Figure 3B-E). 
Current recommendations foresee $10-15 \mathrm{ml}$ of plasma/kg bodyweight every 2-3 weeks ${ }^{3,22,27}$, an interval met in two thirds of patients on prophylaxis in our cohort (Figure 3F). The four patients with the highest annual incidence rates of acute TTP episodes received plasma every 7, 14 days (two cases) and 21 days, respectively (Figure 3F). The mean annual plasma volume normalized to bodyweight was $\leq 400 \mathrm{ml} / \mathrm{kg} /$ year (or $\leq 15 \mathrm{ml} / \mathrm{kg}$ every two weeks) in $79 \%(60 / 76)$ of patients with available information (Figure $3 G)$. The patients with annual plasma volumes of $>400 \mathrm{ml} / \mathrm{kg}$ had annual incidence rates of acute episodes $\leq 1$. During the final weeks of pregnancy, female hTTP patients received the highest doses of plasma that amounted to $30-40 \mathrm{ml} / \mathrm{kg} /$ week (which projects to $\sim 1500-2000 \mathrm{ml} / \mathrm{kg} / \mathrm{year}$ ).

Patients aged $<10$ years at enrollment had the highest incidence rate $(1.18$ [95\% $\mathrm{Cl} 0.88$ 1.55]), which decreased with each decade and was 0.14 [95\% $\mathrm{Cl} 0.08-0.23$ ] in patients aged $>40$ years. Seven of the eight patients with annual incidence rates $>1$ were children between 2 and 13 years of age (Figure 2; patients \#1, 27, 44, 48, 59, 67, and 69; one adult patient \#20; and Figure 3B and C). Our data show that current prophylactic plasma therapy is often insufficient, particularly in children where acute TTP episodes were frequent.

Multivariable analysis considering the effects of age at enrollment, sex, use of prophylaxis and total follow-up time on the annual incidence of TTP episodes confirmed age and further demonstrated that time of follow-up were the main drivers of the annual incidence rates (Table 4). Increasing age reduced the incidence of TTP episodes by a factor of $0.71(95 \% \mathrm{Cl}$ 0.56-0.90) per decade. The apparent higher annual incidence in female patients (Figure 3A) was attributable to their overall younger age (21 years [range 1.2-68] vs. 31 years [range 1.775] in males; $p=0.028)$.

\section{Characteristics, severity and treatment of reported acute TTP episodes during follow-}

\section{up}

The median time between onset of symptoms of an acute episode and start of treatment was 0.0 days (range $0.0-4.0$ days), with $79.4 \%$ (104 of 131) of acute episodes immediately recognized (Table 5). 
Plasma therapy was specifically installed to treat $92 \%$ of acute episodes, also in patients on regular prophylaxis. Treatment of choice was fresh frozen plasma $(n=73)$, or solvent detergent plasma ( $n=34)$, followed by plasma derived factor VIII concentrates $(n=10)$. The remaining 14 episodes were treated with cryo-poor plasma or other blood products (Table 5). Of note, eleven acute episodes were left untreated, as the patients' treating physicians did not see a need for immediate or specific treatment of the episode. Nevertheless, five patients were subsequently, following the episode, started on prophylactic plasma treatment. The median duration of an episode to recovery was 4.0 days (range $0.0-43$ days).

Major clinical presentations during acute episodes were similar between patients on or not on plasma prophylaxis (Table 5). While $90 \%$ of acute episodes in patients not under plasma prophylaxis were triggered by infections, the corresponding number in patients receiving regular plasma therapy was $67 \%(p=0.019)$.

To assess disease severity during follow-up we introduced a score for the acute episodes (Table 1), with the lowest score describing mild episodes, while the highest score 4 was reserved for fatal outcome of typical TTP episodes and/or death from an acute cerebrovascular or cardiovascular event, or maternal death in the context of a pregnancy. During prospective follow-up four patients died (Figure 4A). While the cause of one death remained unknown (and was not categorized as an event) (Figure 2, patient \#62), the other three deaths were considered category 4 events. These three deaths occurred in patients aged 33 years (Figure 2, \#23; cause of death: large cerebral infarction), 39 years (Figure 2, \#38; heart failure) and 56 years (Figure 2, \#81; lethal arrhythmia with asystole during sepsis) who had suffered 12, 5 and 6 acute episodes before enrollment, respectively. Patients \#23 and \#38 were on plasma prophylaxis at the time of death, patient \#81 had stopped regular plasma infusions four months before his death. While patient \#23 had no other TTP episodes during prospective follow-up, patients \#38 and \#81 had both one score-3-event before death. Their calculated annual incidence rates were $0.59,0.34$ and 0.36 , respectively (Figure 4B). Most frequent were acute TTP episodes of severity score $1(102 / 131)$, which occurred primarily in patients with annual incidence rates $>0.5-1$ and $>1$. Ten acute episodes with a 
severity score 3 and the potential of important morbidity and sequelae, were documented in 9 patients (4 females) (Figure 4B), seven of whom had had comorbidities and sequelae at enrollment resulting from score-3-events in the past before enrollment into the Registry. Nine of the ten score-3-events were strokes.

\section{Incidence of acute episodes in relation to ADAMTS13 activity and ADAMTS13 genotype}

To investigate whether residual ADAMTS13 activity at baseline had an impact on the annual incidence rate of acute episodes, we considered only patients $(n=70)$ having their baseline ADAMTS13 activity measured at Bern University Hospital by the modified VWF73-FRETS assay. ${ }^{25,26}$ Because of unknown plasma prophylaxis status we excluded one patient from this analysis. Of the 69 remaining patients, 49 (71\%) had a very severe ADAMTS13 deficiency (ADAMTS13 activity $\leq 1 \%$ ), while 20 had some residual ADAMTS13 activity (between 2$10 \%)$. An annual incidence rate of acute episodes $\leq 0.5$ was seen in $80 \%(16 / 20)$ of patients with residual ADAMTS13 activity at baseline, and in $67.3 \%(33 / 49)$ of patients with an ADAMTS13 activity $\leq 1 \%$ (Figure 3D and E).

A large number of patients in our follow-up study were homozygous ( $n=27)$ or compound heterozygous $(\mathrm{n}=18)$ carriers of the ADAMTS13 c.4143_4144dupA mutation that in homozygotes is associated with no measurable ADAMTS13 activity in the circulation. ${ }^{8}$ The ADAMTS13 c.4143_4144dupA homozygotes were all $\geq 18$ years of age and had a median annual incidence rate of acute episodes of 0.25 (range $0.0-1.02$ ), which was similar to that of the adult patients carrying only one, or no c.4143_4144dupA allele (Supplemental Figure $1 A)$.

Twelve patients (9 females) were compound heterozygous carriers of the ADAMTS13 c.3178C > T (p.R1060W) mutation that is associated with residual ADAMTS13 activity of 3-8\% per allele. ${ }^{6,8}$ Its carriers present often with an adult-onset of overt hTTP, especially during pregnancy. ${ }^{14-16}$ Nine of the compound heterozygous p.R1060W carriers had a low recurrence 
rate (annual incidence rate $\leq 0.5)$; the other three had annual incidence rates of $0.7(n=2)$ and 0.9 , respectively (Supplemental Figure $1 \mathrm{~B}$ ).

In the Registry, there are $13 \mathrm{hTTP}$ patients from six families, who have an affected family member that participates in the Registry (four pairs and one trio of siblings; and one fatherdaughter pair; in Figure 2 brackets link the respective family members). Annual incidence rates of acute episodes between affected siblings was similar in four families and different in the family with three affected siblings and in the father-daughter pair (Supplemental Table 4).

\section{Outcome of pregnancies in hTTP patients during follow-up}

During follow-up, five patients (Figure 2, \#10,12, 16, 76 and 77) had 13 pregnancies; one of them had ended in miscarriage (\#16), four were ongoing at last follow-up visit up to December 2019, and eight pregnancies had resulted in live births of healthy infants who all survived (Supplemental Figure 2). Deliveries were categorized according to the classification recommended by the $\mathrm{WHO}^{28,29}$ for six pregnancies at term (37-41 weeks of gestation), while in two instances delivery was very preterm and late preterm (30 and 36 weeks of gestation, respectively). During the pregnancies, all patients received regular plasma treatment, which had been established before becoming pregnant in three (patients \#10,12, 16), while two women were started on regular plasma infusions immediately when pregnancies were confirmed (\#76, 77). Plasma prophylaxis was closely monitored and volumes adapted with increasing body weight and increased to keep the platelet count in the individual patient's normal range.

\section{DISCUSSION}

Hereditary TTP is an ultra-rare blood disorder with a prevalence of $0.5-2$ cases per million. ${ }^{3}$ Despite increasing awareness over the past two decades, long-term outcome data are largely lacking and available information often stems from retrospective data collections $\mathbf{s}^{5,7,8}$ and single case reports that are a snapshot in time. This is the first large report on 87 hTTP patients prospectively followed in the Hereditary TTP Registry. Over 371 person-years, 44 
patients had no, whereas 43 patients experienced 131 acute TTP episodes corresponding to an overall annual incidence rate of acute episodes of 0.35 (95\% Cl 0.29-0.42; Table 3).

Of the 131 documented acute TTP episodes, 88 (67.2\%) occurred in women, and 75 (57.2\%) in 15 patients aged <18 years at enrollment. Multivariable analysis considering age, sex, use of plasma prophylaxis and time in follow-up demonstrated that not the female sex but age was the driver of the acute episodes. As VWF plasma levels rise with age ${ }^{30}$ and even ADAMTS13 activity values in the lower normal range are associated with an increased risk of ischemic stroke, coronary heart disease and cardiovascular mortality, ${ }^{31,} 32$ we had hypothesized that more acute TTP episodes would be documented in older-aged hTTP patients. On the contrary, we observed a high number of acute events in young patients. Indeed, $35(26.7 \%)$ episodes were documented in seven patients aged 2-7 years, the majority of them occurred in the context of mild to moderate infections (Figure 2B). These observations suggest that early childhood is a vulnerable period in the life of hTTP patients and are in line with recent small case series on pediatric hTTP patients. ${ }^{11,12,17,18}$ Together, this stresses the importance of timely recognition of child-onset hTTP.

An important gap exists between overt disease onset and clinical diagnosis of hTTP that potentially contributed to the high prevalence of comorbidities at enrollment, particularly of arterial thrombotic events. ${ }^{8}$ Despite awareness and an established diagnosis of hTTP in participants of the hereditary TTP Registry as well as regular prophylaxis in the majority of them, the currently employed plasma infusion regimens are insufficient to prevent acute TTP episodes in a significant number of patients. Indeed, while on plasma prophylaxis 31 of 61 hTTP patients experienced 91/131 hTTP episodes (in 254 prospective patient years; annual incidence rate of acute episodes of $0.36 ; 95 \% \mathrm{Cl} 0.29-0.44$, which was only borderline better than without prophylaxis, Table 3). In the majority of patients, regular plasma prophylaxis followed the recommended dose $(10-15 \mathrm{ml} / \mathrm{kg}$ ) and interval (administration every 2-3 weeks) which projects to a plasma volume of $\leq 400 \mathrm{ml} / \mathrm{kg} / \mathrm{year}$. Higher doses were administered to one fifth of patients who all had annual incidence rates of acute episodes $\leq 1$. The highest 
amounts of plasma was given to female hTTP patients during the final weeks of pregnancies with doses as high as $30-40 \mathrm{ml} / \mathrm{kg} /$ week (projecting to $\sim 1500-2000 \mathrm{ml} / \mathrm{kg} / \mathrm{year}$ ). In addition, plasma prophylaxis during pregnancies was closely monitored and adapted to keep the platelet counts in the patient's individual normal range. This approach was effective and associated with delivery at term in $75 \%$ of pregnancies and a live birth rate of $89 \%$, suggesting an improved outcome than previously observed. ${ }^{13-15}$ These are encouraging data and suggest that hTTP patients can have successful pregnancies when regular plasma prophylaxis is installed early, monitored and adapted (e.g. by reducing the interval between administrations, or by increasing the volume given) when necessary. A multidisciplinary approach involving treating physician, hematologist, obstetrician and neonatologist is suggested for the best outcomes. ${ }^{33}$

Chronic ADAMTS13 deficiency as a relevant cardio- and cerebrovascular risk factor ${ }^{31,32}$ is present in all hTTP patients, even in those under strict plasma prophylaxis. Therefore, other existing cardiovascular risk factors should be managed rigorously and long-term treatment with aspirin should be considered. ${ }^{34,35}$

More than 150 different causative ADAMTS13 mutations have been described in hTTP, ADAMTS13 c.4143_4144dupA and ADAMTS13 c.3178C>T (p.R1060W) being the most prevalent. $^{7-9,11,14,15,22}$ Of the 87 hTTP patients in prospective follow-up, 18 carried one, and 27 two ADAMTS13 c.4143_4144dupA alleles, while 12 were carriers of one ADAMTS13 c.3178C >T (p.R1060W) allele. We detected no dosage effect for the number of ADAMTS13 c.4143_4144dupA alleles, although this analysis may have been masked as all but one of the 27 homozygotes were $\geq 18$ years of age at enrollment and thus adults during follow-up (Supplemental Figure 1A). The 12 carriers of one p.R1060W allele had ADAMTS13 activities ranging from $1-9 \%$ and a low incidence rate of acute episodes.

Residual ADAMTS13 activity has been reported to modulate the clinical phenotype in hTTP. 11 Of the 69 hTTP patients with information on plasma prophylaxis and a baseline ADAMTS13 activity determined by the modified FRETS-VWF73 assay, ${ }^{25,}{ }^{26}$ residual baseline 
ADAMTS13 activity (range 2-10\%) was documented in 20 patients, while 49 had a very severe ADAMTS13 deficiency ( $\leq 1 \%)$ (Figure 3D and E). An annual incidence rate of acute episodes of $\leq 0.5$ was observed in $80 \%(16 / 20)$ of patients with residual ADAMTS13 activity at baseline compared to $67.3 \%(33 / 49)$ of patients having an ADAMTS13 activity $\leq 1 \%$. This suggests at most a mild effect of residual baseline ADAMTS13 activity on the clinical phenotype. In two thirds of all episodes a potential trigger was identified, which was in $75 \%$ of cases an infection.

The clinical presentation of acute TTP episodes in our cohort of $87 \mathrm{hTTP}$ patients was variable but produced the most common signs and symptoms published in the literature and ranged from laboratory abnormalities only to clinical manifestations that produced lasting sequelae and three deaths. Presentations were similar in patients with or without plasma prophylaxis (Table 5).

In the past, there have been attempts to assess the severity of hTTP based on accumulated comorbidities and/or special events experienced (i.e. exchange blood transfusion in the neonatal period) $6,8,9,11$ which proved not suitable for the prospective evaluation of hTTP patients. We have developed a scoring system (Table 1) and assessed the severity of all 131 acute hTTP episodes. Most frequent were episodes (102/131) of severity score 1 which occurred primarily in patients with annual incidence rates of $>0.5$ (Figure 4B). Given their temporary effect, they have often not urged the treating physician to change overall treatment. We observed no typical TTP episode with fatal outcome; however, three of the four deaths during follow-up were due to premature cerebrovascular or cardiovascular events (Figure 4A).

Our study has several limitations. Forty-four patients enrolled in the Registry from Japan had to be temporarily excluded from the analysis because of new consent requirements (Figure 1). 
Next, the definition of an acute TTP episode as an illness for which the patient required and/or received medical care in addition to regular prophylactic treatment may lead to underreporting of acute episodes. Many hTTP patients are familiar with ambiguous symptoms such as headaches, irritation, difficulties to concentrate, abdominal discomfort, sub-febrile temperatures, etc. that resolve within a few hours after plasma infusion and are thus an indication of non-overt TTP episodes. Patients have grown accustomed to these ailments and seldom seek medical care. We have no possibility to document all these nonovert or smoldering TTP episodes, which are also difficult to distinguish from headaches or abdominal discomfort of other reasons. We have restricted disease activity to acute overt TTP episodes and accept to underestimate the true burden of the disease. Moreover, the very variable clinical presentation in terms of signs and symptoms as well as in terms of their duration gives the treating physicians a great leeway to interpret and document the findings. There are no evidence-based recommendations or guidelines when to start or how to perform prophylactic treatment in hTTP patients. In case of Registry patients, the treating physicians decide on the initiation and the modalities of prophylaxis. This potentially creates a site-wise selection bias in patients on prophylaxis. Moreover, the documented prophylaxis regimens were variable between different patients but also in a number of patients over time. Our data set is not yet sufficiently large to analyze the different prophylaxis regimens in depth. As the study is ongoing, the ever-growing prospective follow-up will increase robustness of data in the future.

In conclusion, the Hereditary TTP Registry has provided for the first time prospective data of 87 confirmed hTTP patients. The overall annual incidence rate of acute episodes was considerably lower than the annual bleeding rate in patients with hemophilia, ${ }^{36,37}$ another inherited disorder originally treated with plasma infusions. This needs to be taken into account when designing prophylaxis and treatment studies with recombinant ADAMTS13. 
The efficacy of currently employed prophylaxis regimens (infusion of $10-15 \mathrm{ml}$ plasma/kg every 2-3 weeks) is often insufficient to prevent acute episodes. This was particularly true for young children and women. Once recombinant ADAMTS13 becomes available ${ }^{38}$ and regular home treatment will become possible, the decision to treat hTTP patients prophylactically will be less difficult and applied doses will likely be more efficient than currently volume-wise limited plasma regimens. 


\section{AUTHOR CONTRIBUTIONS}

$\mathrm{ET}$, LB, BL, JAKH designed the study, which was approved according to the registry project guidelines by KDF, JNG, IH, PNK, MM, ASVK, BL and JAKH. ET, IAH, and KAJ collected data. ET, LB, BL and JAKH analyzed data. ET and JAKH wrote the paper and had editorial support from JNG and BL. All authors edited the final manuscript.

\section{ACKNOWLEDGMENT}

We thank the many sites and patients from all over the world who participate in this project. The responsible physicians and academics at the different sites participating in this project are listed in Supplemental Material, Appendix A. The contribution of Marienn Réti, Department of Hematology and Stem Cell Transplantation, National Institute of Hematology and Infectious Diseases, Central Hospital of Southern Pest, Budapest, Hungary; of Renata Tomaszewska and Maria Szczepanska, Medical University of Silesia in Katowice, Zabrze, Poland; of Beata Baran, Edyta Odnoczko and Ksenia Bykowska, Institute of Hematology and Transfusion Medicine, Warsaw, Poland in laboratory investigation and/or patient care is gratefully acknowledged.

The hereditary TTP Registry has received support through grants from the Swiss National Science Foundation (grant 310030-185233), the Mach-Gaensslen Foundation Switzerland, the ISTH 2007 Presidential Fund, the GTH Congress President Fund, the NFG Foundation, as well as a research grant from Baxalta US Inc., member of the Takeda group of companies, Bannockburn, IL, USA.

Furthermore, this work was supported in part by grants-in-aid from the Ministry of Health, Labor and Welfare of Japan, and the Higher Education Institutional Excellence Program of the Ministry of Human Capacities in Hungary within the framework of the molecular biology thematic program of the Semmelweis University, Budapest, Hungary. 


\section{DISCLOSURE OF CONFLICT OF INTEREST}

PK received consultancy and advisory board fees, speaker honoraria and travel grants from Ablynx/Sanofi, Alexion, Shire/Takeda.

M.M. is a member of advisory board of Takeda, Alexion, and Sanofi.

A.S. v. K. is a member of advisory board of Sanofi Genzyme

J.W. received grant support or lectures honoraria from Alexion, Alnylam Pharmaceuticals, Baxalta, CSL Behring, Ferring Pharmaceuticals, Novo Nordisk, Octapharma, Rigel Pharmaceuticals, Roche, Sanofi/ Genzyme, Shire/Takeda, Sobi, Werfen.

B.L. is chairman of the data safety monitoring committees for the Baxalta 281102 study (rADAMTS13 in congenital TTP) and for the Takeda SHP655-201 study (rADAMTS13 in acquired TTP), now both run by Takeda. He is a member of the advisory board of Ablynx, now part of Sanofi, for the development of caplacizumab. He received congress travel support and/or lecture fees from Baxter, Ablynx, Alexion, Siemens, Bayer, Roche and Sanofi. J.A.K.H. is a member of the advisory board of Shire, member of the Takeda group of companies for the development of rADAMTS13; and of Ablynx, now part of Sanofi for the development of caplacizumab.

The other authors have nothing to disclose.

\section{DATA SHARING}

For original data, please email the corresponding author. 


\section{REFERENCES}

1. Sadler JE. Pathophysiology of thrombotic thrombocytopenic purpura. Blood. 2017;130:1181-8.

2. Joly BS, Coppo P, Veyradier A. Thrombotic thrombocytopenic purpura. Blood. 2017;129:2836-46.

3. Kremer Hovinga JA, George JN. Hereditary Thrombotic Thrombocytopenic Purpura. N Engl J Med. 2019;381:1653-62.

4. Levy GG, Nichols WC, Lian EC, Foroud T, et al. Mutations in a member of the ADAMTS gene family cause thrombotic thrombocytopenic purpura. Nature. 2001;413:488-94.

5. Fujimura $Y$, Matsumoto $M$, Isonishi A, Yagi $H$, et al. Natural history of Upshaw-Schulman syndrome based on ADAMTS13 gene analysis in Japan. J Thromb Haemost. 2011;9 Suppl 1:283-301.

6. Lotta LA, Wu HM, Mackie IJ, Noris M, et al. Residual plasmatic activity of ADAMTS13 is correlated with phenotype severity in congenital thrombotic thrombocytopenic purpura. Blood. 2012;120:440-8.

7. Alwan F, Vendramin C, Liesner R, Clark A, et al. Characterization and treatment of congenital thrombotic thrombocytopenic purpura. Blood. 2019;133:1644-51.

8. van Dorland HA, Mansouri Taleghani M, Sakai K, Friedman KD, et al. The International Hereditary Thrombotic Thrombocytopenic Purpura Registry: key findings at enrollment until 2017. Haematologica. 2019;104:2107-15.

9. von Krogh AS, Quist-Paulsen P, Waage A, Langseth OO, et al. High prevalence of hereditary thrombotic thrombocytopenic purpura in central Norway: from clinical observation to evidence. J Thromb Haemost. 2016;14:73-82.

10. Furlan M, Lämmle B. Aetiology and pathogenesis of thrombotic thrombocytopenic purpura and haemolytic uraemic syndrome: the role of von Willebrand factor-cleaving protease. Best Pract Res Clin Haematol. 2001;14:437-54.

11. Hassenpflug WA, Obser T, Bode J, Oyen F, et al. Genetic and Functional Characterization of ADAMTS13 Variants in a Patient Cohort with Upshaw-Schulman Syndrome Investigated in Germany. Thromb Haemost. 2018;118:709-22.

12. Joly BS, Coppo P, Veyradier A. Pediatric thrombotic thrombocytopenic purpura. Eur J Haematol. 2018;101:425-34.

13. Fujimura $\mathrm{Y}$, Matsumoto $\mathrm{M}$, Kokame $\mathrm{K}$, Isonishi $\mathrm{A}$, et al. Pregnancy-induced thrombocytopenia and TTP, and the risk of fetal death, in Upshaw-Schulman syndrome: a series of 15 pregnancies in 9 genotyped patients. Br J Haematol. 2009;144:742-54.

14. Moatti-Cohen M, Garrec C, Wolf M, Boisseau P, et al. Unexpected frequency of UpshawSchulman syndrome in pregnancy-onset thrombotic thrombocytopenic purpura. Blood. 2012;119:5888-97.

15. Scully $M$, Thomas $M$, Underwood $M$, Watson $H$, et al. Thrombotic thrombocytopenic purpura and pregnancy: presentation, management, and subsequent pregnancy outcomes. Blood. 2014;124:211-9.

16. von Krogh AS, Kremer Hovinga JA, Tjonnfjord GE, Ringen IM, et al. The impact of congenital thrombotic thrombocytopenic purpura on pregnancy complications. Thromb Haemost. 2014;111:1180-3.

17. Lehmberg K, Hassenpflug WA, Klaassen I, Hillebrand G, et al. Inherited Thrombotic Thrombocytopenic Purpura (Upshaw Schulman Syndrome) as Differential Diagnosis to Neonatal Septicaemia with Disseminated Intravascular Coagulation - a Case Series. Z Geburtshilfe Neonatol. 2017;221:39-42.

18. Fujimura Y, Lämmle B, Tanabe S, Sakai K, et al. Patent ductus arteriosus generates neonatal hemolytic jaundice with thrombocytopenia in Upshaw-Schulman syndrome. Blood Adv. 2019;3:3191-5.

19. Veyradier A, Meyer D, Loirat C. Desmopressin, an unexpected link between nocturnal enuresis and inherited thrombotic thrombocytopenic purpura (Upshaw-Schulman syndrome). J Thromb Haemost. 2006;4:700-1.

20. Borogovac A, George JN. Stroke and myocardial infarction in hereditary thrombotic thrombocytopenic purpura: similarities to sickle cell anemia. Blood Adv. 2019;3:3973-6. 
21. Barbot J, Costa E, Guerra M, Barreirinho MS, et al. Ten years of prophylactic treatment with fresh-frozen plasma in a child with chronic relapsing thrombotic thrombocytopenic purpura as a result of a congenital deficiency of von Willebrand factor-cleaving protease. $\mathrm{Br} \mathrm{J}$ Haematol. 2001;113:649-51.

22. Kovarova P, Hrdlickova R, Blahutova S, Cermakova Z. ADAMTS13 kinetics after therapeutic plasma exchange and plasma infusion in patients with Upshaw-Schulman syndrome. J Clin Apher. 2019;34:13-20.

23. Mansouri Taleghani $M$, von Krogh AS, Fujimura $Y$, George JN, et al. Hereditary thrombotic thrombocytopenic purpura and the hereditary TTP registry. Hamostaseologie. 2013;33:138-43.

24. Scully M, Cataland S, Coppo P, de la Rubia J, et al. Consensus on the standardization of terminology in thrombotic thrombocytopenic purpura and related thrombotic microangiopathies. J Thromb Haemost. 2017;15:312-22.

25. Froehlich-Zahnd R, George JN, Vesely SK, Terrell DR, et al. Evidence for a role of antiADAMTS13 autoantibodies despite normal ADAMTS13 activity in recurrent thrombotic thrombocytopenic purpura. Haematologica. 2012;97:297-303.

26. Kokame K, Nobe Y, Kokubo Y, Okayama A, et al. FRETS-VWF73, a first fluorogenic substrate for ADAMTS13 assay. Br J Haematol. 2005;129:93-100.

27. Scully M, Hunt BJ, Benjamin S, Liesner R, et al. Guidelines on the diagnosis and management of thrombotic thrombocytopenic purpura and other thrombotic microangiopathies. Br J Haematol. 2012;158:323-35.

28. WHO: recommended definitions, terminology and format for statistical tables related to the perinatal period and use of a new certificate for cause of perinatal deaths. Modifications recommended by FIGO as amended October 14, 1976. Acta Obstet Gynecol Scand. 1977;56:247-53.

29. Blencowe H, Cousens S, Oestergaard MZ, Chou D, et al. National, regional, and worldwide estimates of preterm birth rates in the year 2010 with time trends since 1990 for selected countries: a systematic analysis and implications. Lancet. 2012;379:2162-72.

30. Laffan M. Can you grow out of von Willebrand disease? Haemophilia. 2017;23:807-9.

31. Sonneveld MA, de Maat MP, Portegies ML, Kavousi M, et al. Low ADAMTS13 activity is associated with an increased risk of ischemic stroke. Blood. 2015;126:2739-46.

32. Sonneveld MA, Franco OH, Ikram MA, Hofman A, et al. Von Willebrand Factor, ADAMTS13, and the Risk of Mortality: The Rotterdam Study. Arterioscler Thromb Vasc Biol. 2016;36:2446-51.

33. Neave L, Scully M. Microangiopathic Hemolytic Anemia in Pregnancy. Transfus Med Rev. 2018;32:230-6.

34. Arps K, Pallazola VA, Cardoso R, Meyer J, et al. Clinician's Guide to the Updated ABCs of Cardiovascular Disease Prevention: A Review Part 1. Am J Med. 2019;132:e569-e80.

35. Arps K, Pallazola VA, Cardoso R, Meyer J, et al. Clinician's Guide to the Updated ABCs of Cardiovascular Disease Prevention: A Review Part 2. Am J Med. 2019;132:e599-e609.

36. Manco-Johnson MJ, Abshire TC, Shapiro AD, Riske B, et al. Prophylaxis versus episodic treatment to prevent joint disease in boys with severe hemophilia. $\mathrm{N}$ Engl $\mathrm{J}$ Med. 2007;357:535-44.

37. Oldenburg J. Optimal treatment strategies for hemophilia: achievements and limitations of current prophylactic regimens. Blood. 2015;125:2038-44.

38. Scully M, Knöbl P, Kentouche K, Rice L, et al. Recombinant ADAMTS-13: first-in-human pharmacokinetics and safety in congenital thrombotic thrombocytopenic purpura. Blood. 2017;130:2055-63. 
Table 1.

Definition of acute hTTP episodes and grading system to assess their severity.

\begin{tabular}{|c|c|}
\hline Acute hTTP episodes & $\begin{array}{l}\text { A typical TTP episode }{ }^{24} \text {, or an acute cerebrovascular or cardiovascular or } \\
\text { other ischemic event; or a renal illness for which the patient requires } \\
\text { and/or receives medical care in addition to regular prophylactic treatment, } \\
\text { e.g. with plasma infusions. } \\
\text { In addition, maternal pregnancy complications resulting in a premature } \\
\text { termination of pregnacy by abortion or delivery after } 10 \text { and before } 34 \\
\text { weeks of gestation. } \\
\text { If laboratory assays are performed, they typically show: } \\
\text { - thrombocytopenia } \\
\text { - presence of schistocytes on peripheral blood smear } \\
\text { - decreased hemoglobin, or reduced hematocrit } \\
\text { - decreased haptoglobin } \\
\text { - increased LDH } \\
\text { - increased (indirect) bilirubin } \\
\text { - increased reticulocyte count }\end{array}$ \\
\hline $\begin{array}{l}\text { Mild episode }= \\
\text { severity score } 1\end{array}$ & $\begin{array}{l}\text { Typical signs and symptoms: } \\
\text { - } \quad \text { petechiae, ecchymosis/bruises, small hematomas } \\
\text { - } \quad \text { fatigue, drowsiness, weakness, } \\
\text { - } \quad \text { (sub)-febrile temperatures, shivering } \\
\text { - } \text { gastrointestinal symptoms (e.g. vomiting, abdominal discomfort, } \\
\text { diarrhea) } \\
\text { - } \quad \text { hicro- or macro-hematuria } \\
\text { - tachycardia, moderate dyspnea }\end{array}$ \\
\hline $\begin{array}{l}\text { Moderate episode = } \\
\text { severity score } 2\end{array}$ & $\begin{array}{l}\text { Typical signs and symptoms: } \\
\text { - } \quad \text { transient ischemic attack (TIA) } \\
\text { - } \quad \text { angina pectoris } \\
\text { - } \quad \text { cardiac arrhythmia } \\
\text { - } \quad \text { pregnance renal failure, not requiring renal replacement } \\
\quad \text { gestation }\end{array}$ \\
\hline $\begin{array}{l}\text { Severe episode }= \\
\text { severity score } 3\end{array}$ & $\begin{array}{l}\text { Typical signs and symptoms: } \\
\text { - } \quad \text { ischemic stroke } \\
\text { - coma, seizure or impaired conciousness requiring airway protective } \\
\text { - } \text { measures and ventilation } \\
\text { - } \quad \text { acute myocardial infarction } \\
\text { - multi-organ failure } \\
\text { - } \quad \text { pregnancy with abortion } \geq 10^{\text {th }} \text { week of gestation, or a premature } \\
\quad \text { delivery }<34^{\text {th }} \text { week of gestation (maternal indication) }\end{array}$ \\
\hline $\begin{array}{l}\text { Fatal episode }= \\
\text { severity score } 4\end{array}$ & $\begin{array}{l}\text { Fatal outcome of a typical TTP episode and/or death from a cerebro- } \\
\text { vascular or cardiovascular event, or death during pregnancy }\end{array}$ \\
\hline
\end{tabular}


Table 2. Baseline demographic and clinical characteristics of $87 \mathrm{hTTP}$ patients, including 43 with and 44 without acute episodes during follow-up.

\begin{tabular}{|c|c|c|c|c|c|c|c|}
\hline & $\mathrm{N}$ & $\begin{array}{c}\text { All patients } \\
\text { median [min, lq, uq, max }{ }^{*} \\
\text { or } \mathrm{n}(\%)\end{array}$ & $\mathrm{N}$ & $\begin{array}{l}\text { No TTP episodes during } \\
\text { follow-up } \\
\text { median [min, lq, uq, max] } \\
\text { or } \mathrm{n}(\%)\end{array}$ & $\mathrm{N}$ & $\begin{array}{c}\text { Acute TTP episodes during } \\
\text { follow-up } \\
\text { median [min, lq, uq, max] } \\
\text { or } \mathrm{n}(\%)\end{array}$ & $\begin{array}{c}\mathrm{P}- \\
\text { value }^{\$}\end{array}$ \\
\hline $\begin{array}{l}\text { Gender } \\
\text { - Male } \\
\text { - Female }\end{array}$ & 87 & $\begin{array}{l}46(53 \%) \\
41(47 \%)\end{array}$ & 44 & $\begin{array}{l}27(61 \%) \\
17(39 \%)\end{array}$ & 43 & $\begin{array}{l}19(44 \%) \\
24(56 \%)\end{array}$ & 0.13 \\
\hline Consanguinity of parents & 67 & $9(13 \%)$ & 32 & $6(19 \%)$ & 35 & $3(8.6 \%)$ & 0.29 \\
\hline Family member is confirmed patient & 87 & $13(15 \%)$ & 44 & $5(11 \%)$ & 43 & $8(19 \%)$ & 0.38 \\
\hline $\begin{array}{l}\text { Age at } \\
\text { - overt disease onset (years) } \\
\text { - clinical diagnosis (years) } \\
\text { - enrollment (years) } \\
\text { - the last follow-up (years) }\end{array}$ & $\begin{array}{l}77 \\
85 \\
87 \\
87\end{array}$ & \begin{tabular}{cc}
\multicolumn{3}{c}{4.6} & {$[0.0,0.0,19,70]$} \\
18 & {$[0.0,4.2,29,70]$} \\
26 & {$[1.2,16,40,75]$} \\
32 & {$[2.1,21,46,79]$}
\end{tabular} & $\begin{array}{l}35 \\
42 \\
44 \\
44\end{array}$ & $\begin{array}{cc}4.7 & {[0.0,0.0,20,70]} \\
20 & {[0.0,9.4,33,70]} \\
27 & {[1.7,18,44,75]} \\
31 & {[5.2,22,48,79]}\end{array}$ & $\begin{array}{l}42 \\
43 \\
43 \\
43\end{array}$ & $\begin{array}{cc}3.6 & {[0.0,0.0,19,68]} \\
15 & {[0.0,3.5,28,68]} \\
26 & {[1.2,14,37,69]} \\
32 & {[2.1,21,42,73]}\end{array}$ & $\begin{array}{l}0.80 \\
0.12 \\
0.33 \\
0.51\end{array}$ \\
\hline $\begin{array}{l}\text { Treatment } \\
\text { - Prophylactic plasma infusions } \\
\text { - Antiaggregation/anticoagulation } \\
\text { - Antihypertensive medication }\end{array}$ & $\begin{array}{l}83 \\
79 \\
79\end{array}$ & $\begin{array}{l}47(57 \%) \\
21(27 \%) \\
25(32 \%)\end{array}$ & $\begin{array}{l}40 \\
37 \\
37\end{array}$ & $\begin{array}{l}24(60 \%) \\
12(32 \%) \\
14(38 \%)\end{array}$ & $\begin{array}{l}43 \\
42 \\
42\end{array}$ & $\begin{array}{r}23(53 \%) \\
9(21 \%) \\
11(26 \%)\end{array}$ & $\begin{array}{l}0.66 \\
0.31 \\
0.33\end{array}$ \\
\hline \multicolumn{8}{|l|}{ Comorbidities at enrollment: } \\
\hline Arterial thrombotic disease & 83 & $30(36 \%)$ & 41 & $19(46 \%)$ & 42 & $11(26 \%)$ & 0.07 \\
\hline $\begin{array}{l}\text { - Myocardial infarction } \\
\text { - Transient ischemic attack (TIA) } \\
\text { - Stroke } \\
\text { - Other }\end{array}$ & $\begin{array}{l}83 \\
83 \\
83 \\
83\end{array}$ & $\begin{array}{c}4(4.8 \%) \\
13(16 \%) \\
22(27 \%) \\
4(4.8 \%)\end{array}$ & $\begin{array}{l}41 \\
41 \\
41 \\
41\end{array}$ & $\begin{array}{c}2(4.9 \%) \\
7(17 \%) \\
15(37 \%) \\
3(7.3 \%)\end{array}$ & $\begin{array}{l}42 \\
42 \\
42 \\
42\end{array}$ & $\begin{array}{l}2(4.8 \%) \\
6(14 \%) \\
7(17 \%) \\
1(2.4 \%)\end{array}$ & $\begin{array}{l}1.00 \\
0.77 \\
0.049 \\
0.36\end{array}$ \\
\hline Neurological disorders & 83 & $18(22 \%)$ & 41 & $13(32 \%)$ & 42 & $5(12 \%)$ & 0.035 \\
\hline $\begin{array}{l}\text { - Epileptic seizure } \\
\text { - Headache } \\
\text { - Other neurological disease }\end{array}$ & $\begin{array}{l}83 \\
83 \\
83\end{array}$ & $\begin{array}{r}6(7.2 \%) \\
3(3.6 \%) \\
12(14 \%)\end{array}$ & $\begin{array}{l}41 \\
41 \\
41\end{array}$ & $\begin{array}{l}6(15 \%) \\
2(4.9 \%) \\
8(20 \%)\end{array}$ & $\begin{array}{l}42 \\
42 \\
42\end{array}$ & $\begin{array}{l}0(0 \%) \\
1(2.4 \%) \\
4(10 \%)\end{array}$ & $\begin{array}{l}0.012 \\
0.62 \\
0.23\end{array}$ \\
\hline Venous thrombotic disease & 83 & $3(3.6 \%)$ & 41 & $3(7.3 \%)$ & 42 & $0(0 \%)$ & 0.12 \\
\hline Renal insufficiency & 83 & $26(31 \%)$ & 41 & $11(27 \%)$ & 42 & $15(36 \%)$ & 0.48 \\
\hline $\begin{array}{l}\text { - Pathological renal parameters } \\
\text { - On dialysis } \\
\text { - Kidney transplantation }\end{array}$ & $\begin{array}{l}83 \\
83 \\
83\end{array}$ & $\begin{array}{r}17(20 \%) \\
8(10 \%) \\
2(2.4 \%)\end{array}$ & $\begin{array}{l}41 \\
41 \\
41\end{array}$ & $\begin{array}{l}7(17 \%) \\
2(4.9 \%) \\
0(0 \%)\end{array}$ & $\begin{array}{l}42 \\
42 \\
42\end{array}$ & $\begin{aligned} & 10(24 \%) \\
& 6(14 \%) \\
& 2(4.8 \%)\end{aligned}$ & $\begin{array}{l}0.59 \\
0.26 \\
0.49\end{array}$ \\
\hline Neonatal hyperbilirubinemia & 83 & $36(43 \%)$ & 41 & $16(39 \%)$ & 42 & $20(48 \%)$ & 0.51 \\
\hline
\end{tabular}


${ }^{*} \mathrm{~N}$ : number of patients with information available; $\mathrm{n}$ : number of patients in each category; min: minimum; max: maximum; lq: lower quartile; uq: upper quartile. \$Comparison of groups with and without acute episodes during follow-up 
Table 3.

Incidence of documented acute episodes during follow-up according to sex, age at enrollment and prophylactic plasma treatment.

\begin{tabular}{|l|c|c|c|c|c|}
\hline & $\begin{array}{c}\text { Patients with } \\
\text { follow-up }\end{array}$ & $\begin{array}{c}\text { Patients with } \\
\text { any episode }\end{array}$ & $\begin{array}{c}\text { Total } \\
\text { prospective } \\
\text { episodes }\end{array}$ & $\begin{array}{c}\text { Total } \\
\text { person- } \\
\text { years }\end{array}$ & $\begin{array}{c}\text { Annual incidence } \\
\text { rate (95\% CI) }\end{array}$ \\
\hline Overall & 87 & 43 & 131 & 371 & $0.35(0.29-0.42)$ \\
Male & 46 & 19 & 43 & 194 & $0.22(0.16-0.30)$ \\
Female & 41 & 24 & 88 & 177 & $0.50(0.40-0.61)$ \\
\hline Age at enrollment (years) & 15 & 9 & 51 & 43 & $1.18(0.88-1.55)$ \\
$<10$ & 16 & 8 & 27 & 78 & $0.35(0.23-0.50)$ \\
$10-20$ & 18 & 8 & 21 & 75 & $0.28(0.17-0.43)$ \\
$20-30$ & 15 & 9 & 17 & 65 & $0.26(0.15-0.42)$ \\
$30-40$ & 23 & 9 & 15 & 110 & $0.14(0.08-0.23)$ \\
$>40$ & 61 & 31 & 91 & 254 & $0.36(0.29-0.44)$ \\
\hline Prophylaxis & & & & & \\
Yes & 40 & 17 & 40 & 97 & $0.41(0.30-0.56)$ \\
\hline No & & & &
\end{tabular}

* For four patients with 21 person-years and 0 episodes data on prophylaxis was not available; 18 patients had follow-up time with and without prophylaxis (5 with episodes). 
Table 4.

Effect of age at enrollment, gender, use of prophylaxis and total follow-up time on the incidence of documented TTP episodes during follow-up. Multivariable analysis using mixed-effects Poisson regression model with robust standard errors. Only patients with known prophylaxis status (83/87 hTTP patients) were included.

\begin{tabular}{|l|c|c|}
\hline & $\begin{array}{c}\text { Incidence rate ratio } \\
(95 \% \mathrm{Cl})\end{array}$ & P-value \\
\hline $\begin{array}{l}\text { Age at enrollment (per decade) } \\
\text { Gender } \\
\text { Male }\end{array}$ & $0.71(0.56-0.90)$ & 0.005 \\
Female & 1 (Reference) & \\
$\begin{array}{l}\text { Under prophylaxis } \\
\text { No } \\
\text { Yes }\end{array}$ & $1.49(0.80-2.77)$ & 0.21 \\
Follow-up time (per year) & $0.72(0.39-1.35)$ & 0.31 \\
\hline
\end{tabular}


Table 5. Specification, clinical presentation and treatment of acute TTP episodes during follow-up.

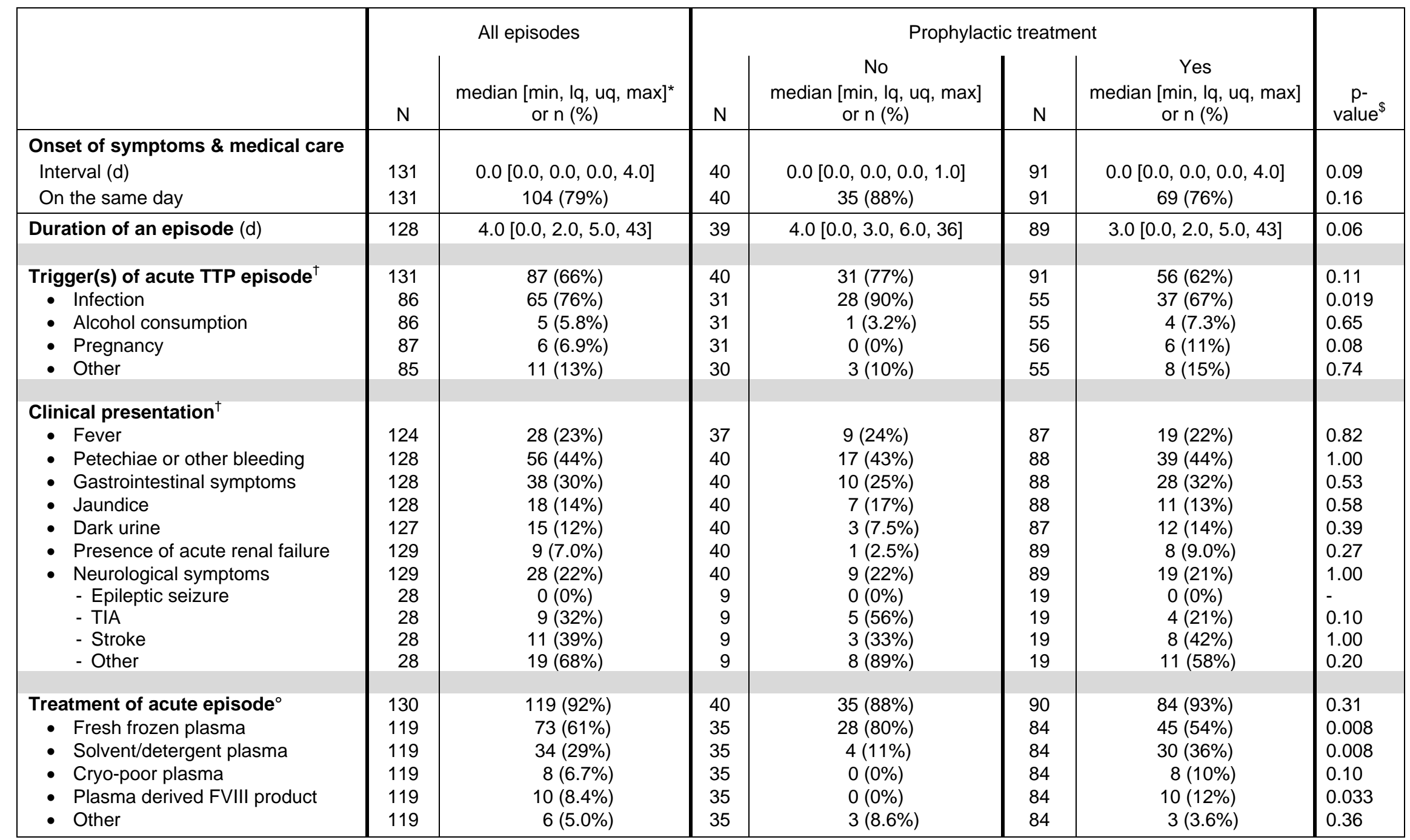


${ }^{*} \mathrm{~N}$ : number of episodes with information available; $\mathrm{n}$ : number of episodes in each category; min: minimum; max: maximum; lq: lower quartile; uq: upper quartile. ${ }^{\$}$ Comparison of groups on and not on prophylactic treatment.

${ }^{\dagger}$ Listings are not additive, multiple entries possible.

- For 11 of the 131 acute episodes patients received no treatment, for one episode no treatment details were documented. 


\section{Legends to Figures}

\section{Figure 1}

\section{Study flow chart.}

Participants in the International Hereditary TTP Registry at the end of 2019 and confirmed hereditary TTP patients followed prospectively.

\footnotetext{
${ }^{*}$ Patients described in van Dorland et al. ${ }^{8}$
}

\section{Figure 2}

Individual disease courses in $87 \mathrm{hTTP}$ patients (46 males and 41 females) after enrollment until last visit up to December 2019.

Panel A. Follow-up (in years) is given on the x-axis. Individual patients are listed on the $y$ axis. Brackets (to the left of the patients' numbers) link patients with affected family members participating in the Registry. Patients are sorted according to sex and use of prophylaxis.

Panel B. Extract of the whole cohort, depicting patients aged $<18$ years at enrollment $(n=25)$, sorted according to age starting with the youngest child. Patients are divided (from uppermost to lowest on the ordinate) in groups of 0 to $<6$ years of age $(n=11), 6$ to $<12$ years of age $(n=6)$, and 12 to $<18$ years of age $(n=8)$.

\section{Figure 3}

Annual incidence of acute TTP episodes in 87 confirmed hTTP patients based on prospective follow-up until their last visit up to end of 2019.

Panel A. Patients grouped according to their annual incidence of acute TTP episodes. While 44 hTTP patients (50.6\%; 27 males, 17 females) experienced no acute episode during follow-up, an annual incidence of $>0$ to 0.5 was documented in 19 (21.8\%; 8 females); of $>0.5$ to 1 in 16 (18.4\%; 11 females); and of $>1$ in 8 (9.2\%; 5 females) hTTP patients.

Panel B and C: Annual incidence of acute TTP episodes in individual patients according to age at enrollment. Patients were under plasma prophylaxis $(B ; n=54)$ or had no plasma prophylaxis $(\mathrm{C} ; \mathrm{n}=29)$ at the last follow-up visit. For four patients, information on plasma prophylaxis was missing.

Panels D and E: Annual incidence of acute TTP episodes in relation to ADAMTS13 activity in 69 hTTP patients having had ADAMTS13 activity measurements by the modified FRETSVWF73 assay. ${ }^{25,26}$ To assess baseline (endogenous) ADAMTS13 activity, blood samples 
were withdrawn at least 14 days after the last administration of plasma-containing blood products. Patients were under plasma prophylaxis (D) or had no plasma prophylaxis (E) at the last follow-up.

Panels F and G: Annual incidence of acute TTP episodes in relation to interval of regular plasma prophylaxis $(F)$ and to the mean dose of plasma administered per kilogram bodyweight and year in 78 hTTP patients with complete datasets for this analysis $(\mathrm{G})$. The two patients regularly treated with a plasma-derived FVIII concentrate are shown separately.

\section{Figure 4}

Severity of acute TTP episodes and survival of hTTP patients during prospective follow-up.

Panel A. Kaplan-Meier curve for overall survival of 87 hTTP patients during prospective follow-up, during which four male patients died. Causes of death were: large cerebral infarction in a 33-year-old; heart failure at age 38 years; lethal arrhythmia with asystole during sepsis at age 56 years, and death of unknown cause at age 79 years. According to the treating physicians, at the time of death none of the patients was considered to have an acute TTP episode. Still, three of the four deaths were categorized as fatal outcome of cerebrovascular or cardiovascular events (acute hTTP episode, severity score 4).

Panel $\mathbf{B}$. The severity of acute TTP episodes are graded as follows (Table 1): score 1 - mild; score 2 - moderate, usually temporary clinical symptoms; score 3 - severe, clinical symptoms producing often permanent morbidity; score 4 - fatal outcome of typical TTP episode and/or death of cerebrovascular or cardiovascular event, or a female patient's death during pregnancy. 


\section{Figure 1}

Participants in Registry ( $N=209)$

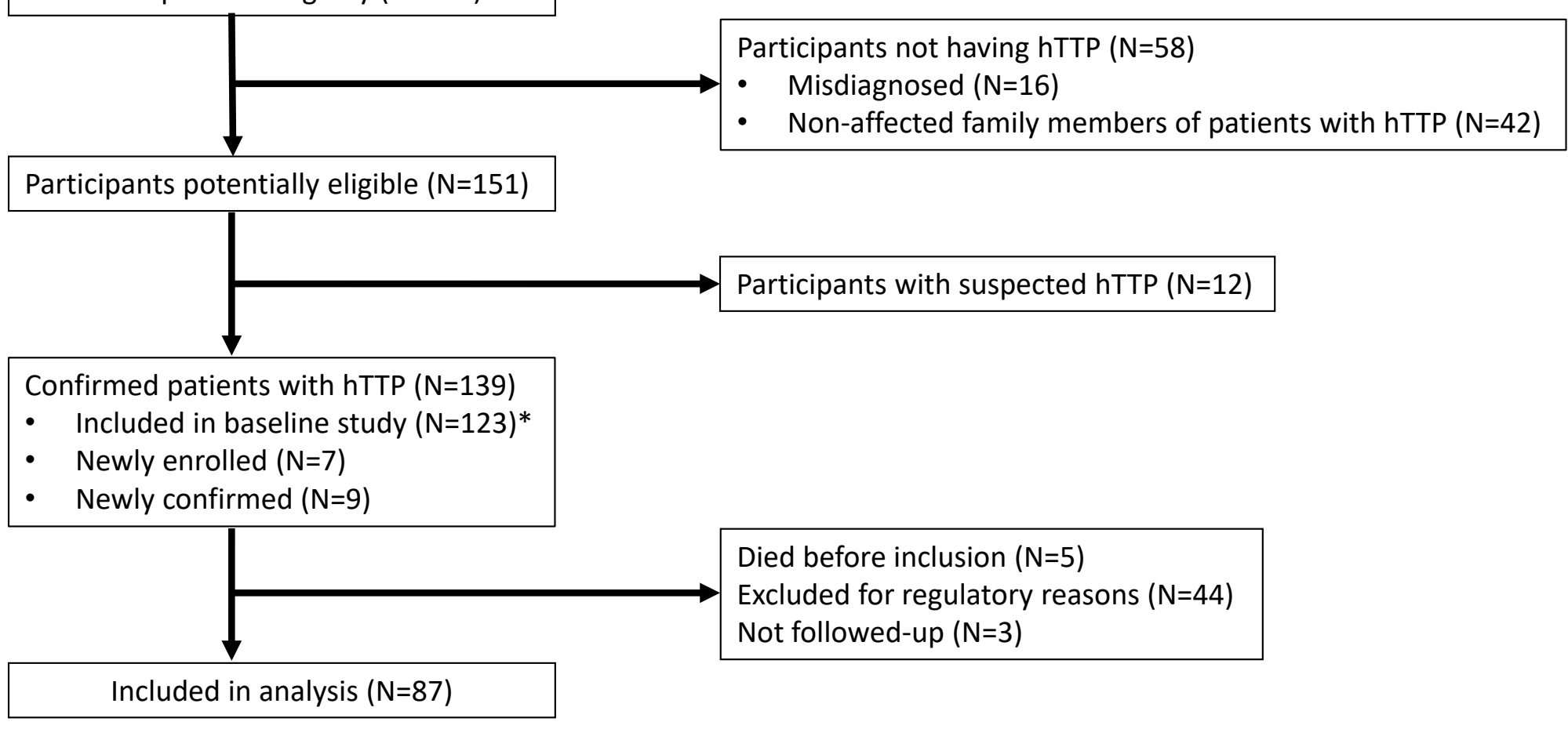


Figure 2

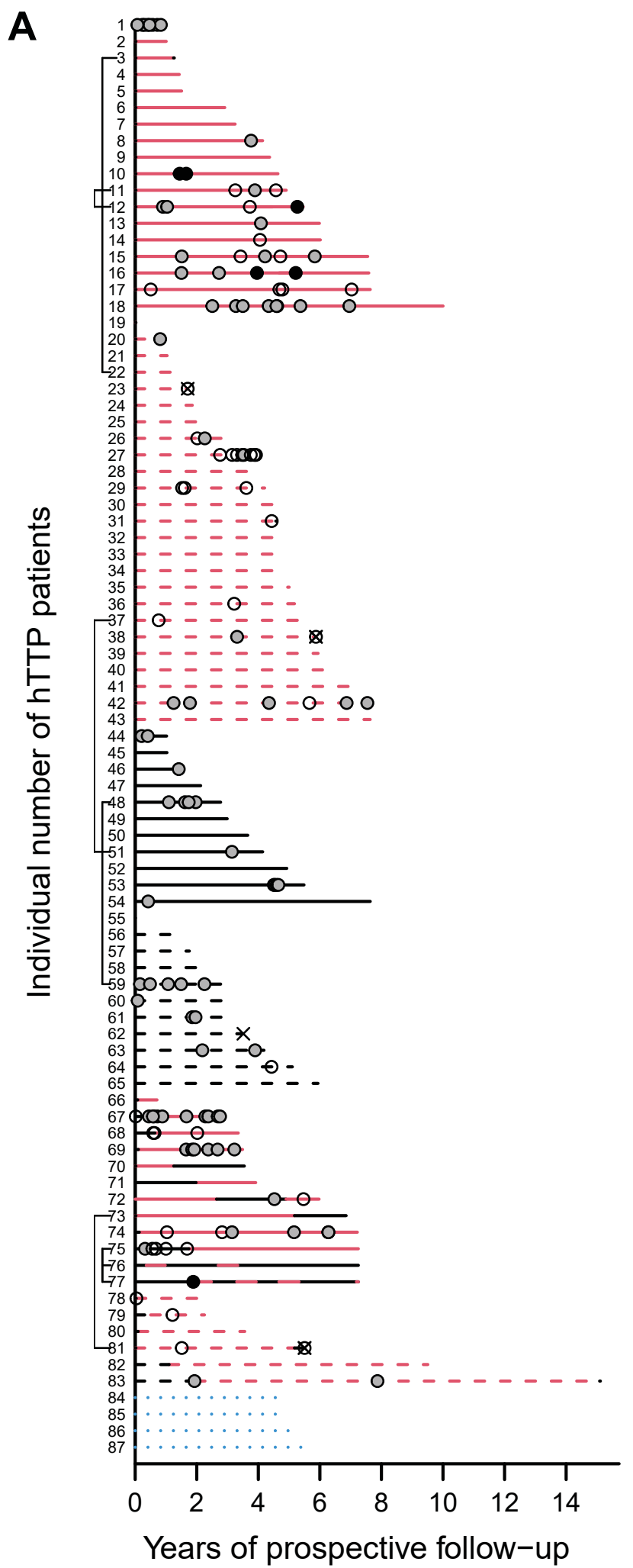

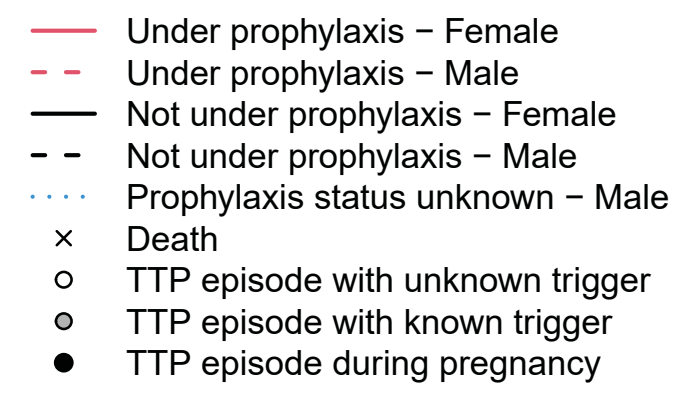

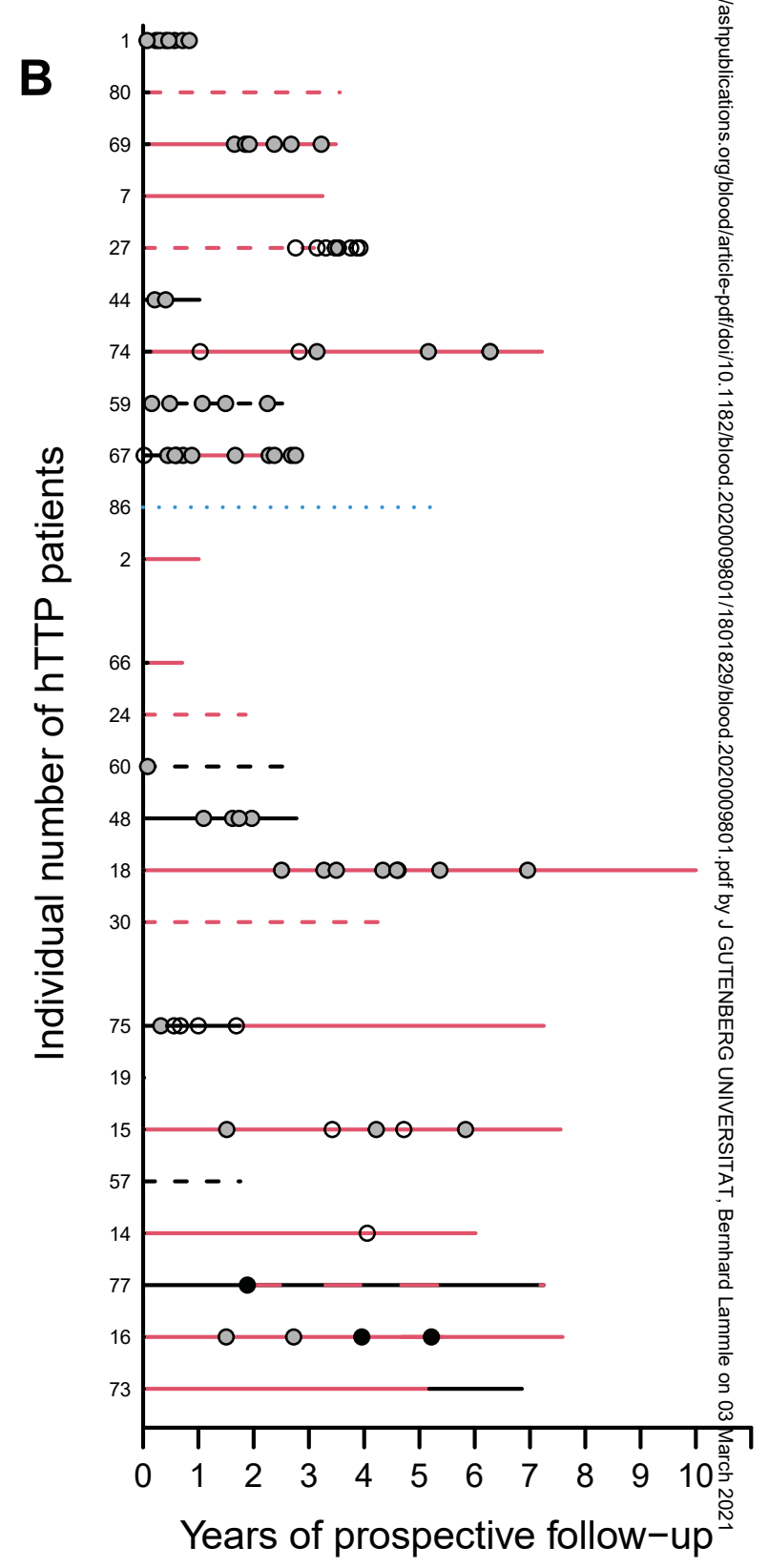


Figure 3
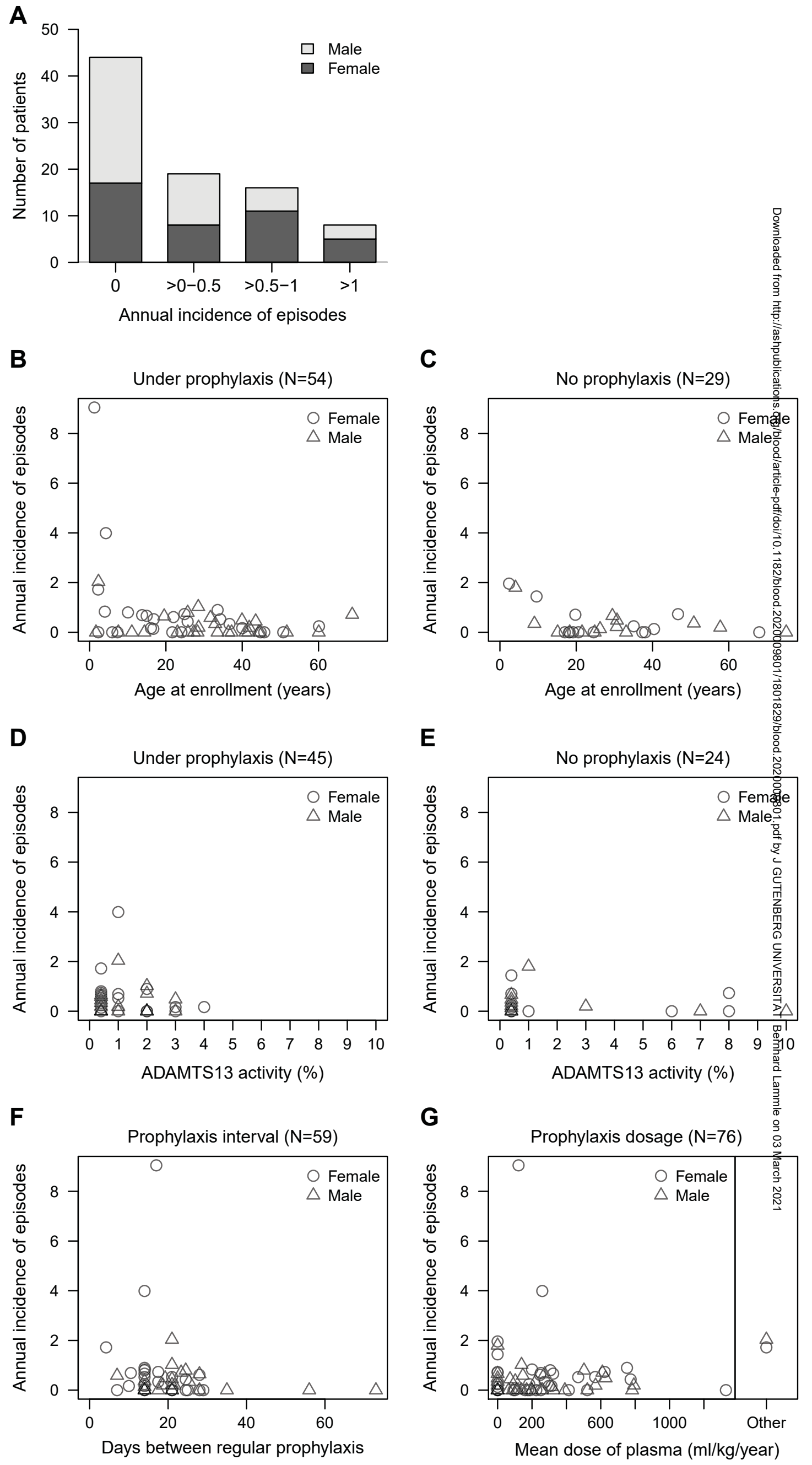
Figure 4

A

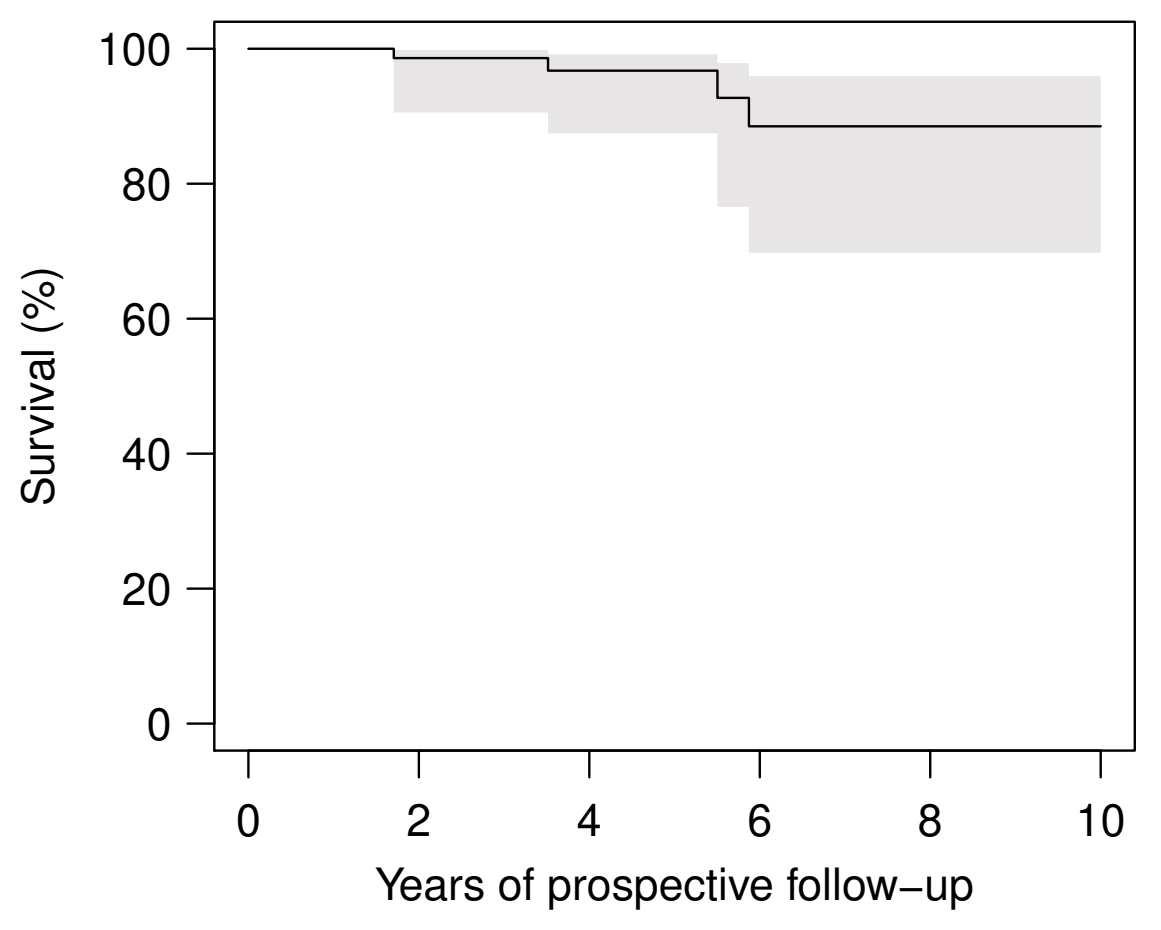

Number at risk 87
B $\frac{0}{8}$

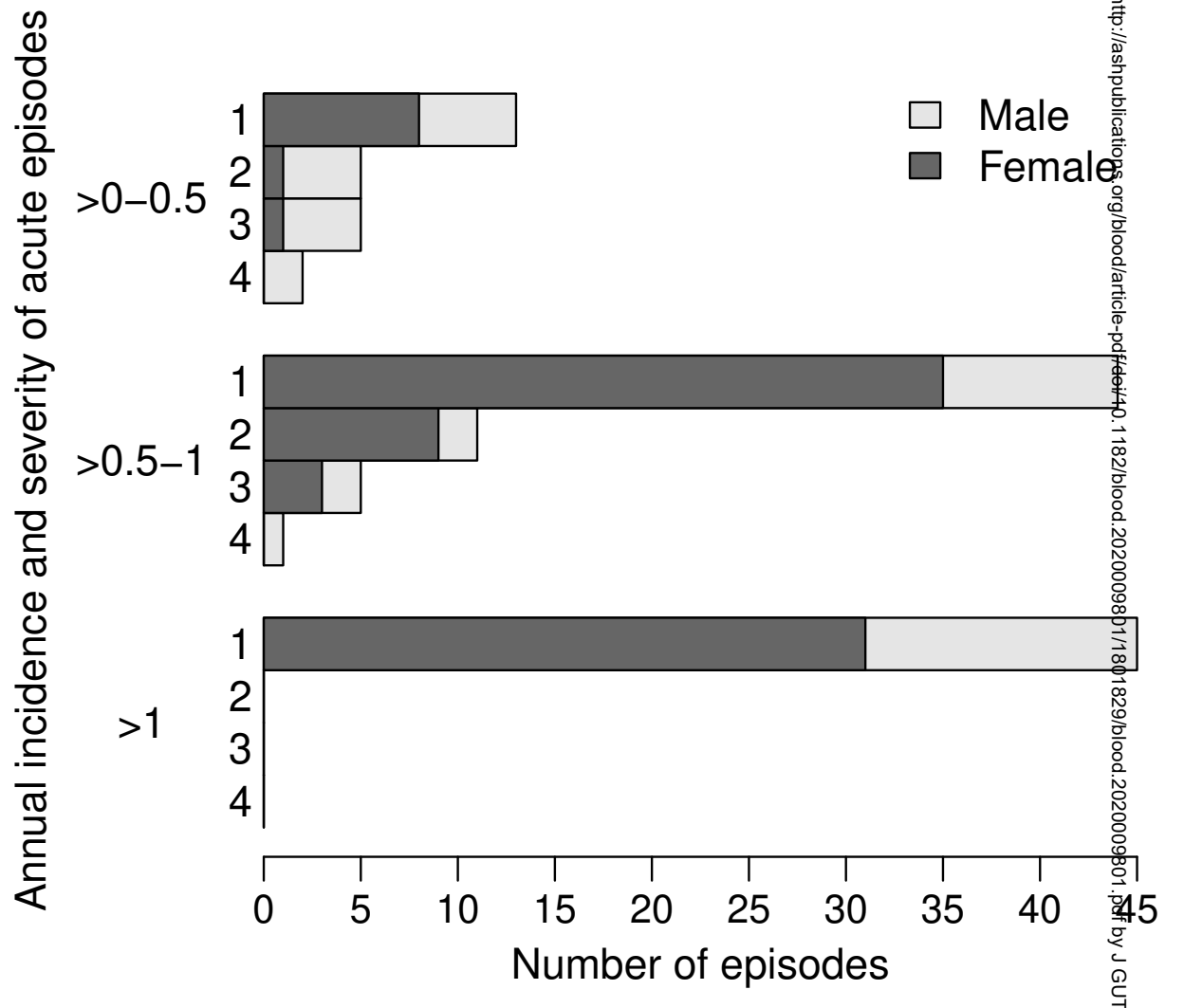

